\section{Uso da Classificação Internacional de Doenças em Inquéritos de Saúde}

\section{Using the International Classification of Diseases in Health Surveys}

Chester Luiz Galvão Cesar

Departamento de Epidemiologia Faculdade de Saúde Pública/USP

Av. Dr. Arnalo, 715

01246-904 São Paulo, SP - Brasil

e-mail: clcesar@usp.br

\section{Ruy Laurenti}

Departamento de Epidemiologia

Faculdade de Saúde Pública/USP

\section{Cássia Maria Buchala}

Departamento de Epidemiologia

Faculdade de Saúde Pública/USP

\section{Gerusa Maria Figueiredo}

Secretaria de Estado da Saúde de São Paulo

\section{Wladithe Organ de Carvalho}

Departamento de Epidemiologia

Faculdade de Saúde Pública/USP

Aluna de pós-graduação

\section{Carla V. de S. Caratin}

Departamento de Nutrição

Faculdade de Saúde Pública/USP

Aluna de pós-graduação

\section{Resumo}

O objetivo do trabalho foi descrever a morbidade "referida" em inquérito de saúde, comparando o uso da CID-9 com o da CID-10. O estudo foi realizado com uma amostra populacional de 10.199 indivíduos, identificando-se as doenças ou os acidentes ocorridos num período de quinze dias. Realizou-se também uma comparação entre a CID-9 e a CID-10, reclassificando-se pela CID-10 as morbidades classificadas pela CID9 como Capítulo XVI (Sintomas, Sinais e Afecções Mal Definidas). Cerca de 30\% dos entrevistados referiu algum problema de saúde, somando 4415 queixas ou diagnósticos. Um total de 1403 queixas foram classificadas, pela CID-9, em 51 códigos do Capítulo XVI. Estas mesmas queixas recodificadas pela CID-10 transformaram-se em 70 Códigos, ganhando maior especifididade. Concluiu-se que foi satisfatória a classificação das morbidades referidas pelos entrevistados por meio da CID, sendo que a CID-10 apresentou vantagens em relação à CID-9, possibilitando classificar subcategorias residuais ao longo dos diferentes capítulos e apresentado maior especificidade do Capítulo XVIII.

Palavras-chave: Classificação Internacional de Doenças. Levantamentos epidemiológicos. Morbidade. Inquéritos de morbidade. 


\section{Abstract}

The objective of the study was to describe morbidity "referred" in health surveys, comparing ICD-9 and ICD-10. The study identified the conditions or injuries occurring within a 15-day period in a population sample of 10,199 individuals. ICD-9 and ICD-10 were also compared, by reclassifying conditions formerly classified as ICD-9 Chapter XVI (Symptoms, Signs and Ill-Defined Conditions) using ICD-10. Approximately $30 \%$ of interviewees referred some health condition, totaling 4,415 complaints or diagnoses. A total of 1,403 complaints were classified, by ICD-9, into 51 Chapter XVI codes. The same complaints, recoded by ICD-10, turned into 70 Codes, therefore gaining greater specificity. The conclusion is that the classification of morbidity referred by interviewees using ICD was satisfactory. ICD10 had advantages in relation to ICD-9, once it enabled the classification of residual subcategories in various chapters, thus providing greater specificity in Chapter XVIII.

Keywords: International classification of diseases. Health surveys. Morbidity. Morbidity surveys.

\section{Introdução}

A interface entre a Epidemiologia e os Serviços de Saúde vem apresentando uma importância crescente na gerência dos serviços de saúde, com a articulação entre estas duas áreas ocorrendo em diferentes etapas do processo administrativo, desde a análise da situação de saúde, planejamento das ações até a avaliação do impacto das atividades desenvolvidas. Há algumas décadas diversos países passaram a realizar periodicamente inquéritos de saúde de base populacional, onde são coletadas simultaneamente informações sobre a situação de saúde, as condições de vida e a utilização dos serviços de saúde. Os bancos de dados criados a partir destes inquéritos possibilitam uma análise mais abrangente dos serviços de saúde ao incorporarem informações sobre a população atendida e não atendida pelos serviços, permitindo até a caracterização desta demanda, segundo diferentes variáveis como sexo, idade, local de moradia e condições socioeconômicas.

Com alguma freqüência estes inquéritos coletam dados sobre a morbidade "referida" pelos entrevistados que é, geralmente, codificada pela Classificação Internacional de Doenças - CID para as análises. Alguns autores têm considerado a CID como inadequada, tanto para a codificação dos diagnósticos na atenção primária à saúde como nos inquéritos de morbidade. O principal argumento é de que a CID é muito detalhada para codificar "queixas" que freqüentemente são muito genéricas, embora admitam que, apesar de existirem classificações mais próximas à realidade dos inquéritos de saúde, a CID permite maior comparabilidade com outras fontes rotineiras de informação ${ }^{1-4}$.

A CID teve início em 1893 como instrumento estatístico para enumerar e analisar as causas de morte sem incluir as doenças não mortais, sendo que em 1900 foi realizada em Paris a $1^{\text {a }}$ Conferência Internacional para revisar a Classificação Internacional de Causas de Morte, como era então conhecida $^{5}$. A partir da $6^{\text {a }}$ Revisão, de 1948, a res- 
ponsabilidade das revisões passou a ser da OMS e foi discutida a possibilidade de duas classificações: uma para mortalidade, seu uso tradicional, e outra para morbidade. Optouse por manter apenas uma só CID, a qual foi expandida a fim de adequar-se à morbidade, estabelecendo-se assim uma Lista de Causas de Morbidade ${ }^{5-8}$.

No entanto, foram desenvolvidas diversas classificações, em diferentes países, na busca de uma específica e adequada para morbidade em assistência básica, sendo que todas elas mantiveram alguma ligação com a CID. Além de problemas relativos à sua validade e reprodutibilidade, estas classificações não satisfizeram completamente às necessidades existentes ${ }^{1-4,7,9-13}$. Uma delas, a Classificação Internacional para Assistência Primária (CIAP), foi testada no Brasil, com a conclusão de viabilidade do seu uso. Mesmo sendo uma classificação específica, o Capítulo de Problemas Gerais e Inespecíficos agrupou a maior proporção de motivos de consulta $^{14}$.

A 9a e a $10^{a}$ Revisões da CID $^{5,15,16}$ foram bastantes expandidas com vistas à morbidade. Na 9a revisão conservou-se a estrutura básica da CID, com detalhes e algumas subdivisões opcionais para possibilitar especificações ou manifestações de uma mesma doença. Além disto, passou a ter "dupla classificação” para muitas doenças, com um código $(*)$ para manifestações e outro $(\boldsymbol{\dagger})$ para mortalidade, concessão feita para que fosse possível utilizá-la para morbidade ${ }^{3,4,8}$.

A $10^{\text {a }}$ Revisão expandiu mais a CID, aumentando o número de códigos para uso em morbidade, incorporando mais categorias e especificações na classificação de motivos de consulta que não constituem doenças ou lesões.

A classificação simplificada de três caracteres existente na CID-10 foi considerada o "núcleo" de uma "família de classificações" e a ele somam-se outras classificações relacionadas hierarquicamente ou de natureza suplementar ${ }^{3,4,16,17}$. A "família” consegue atingir diferentes necessidades e usos em saúde pública, inclusive estatísticas de motivos de consulta em assistência primária.
Assim, a CID, inicialmente usada somente para mortalidade, foi expandida a fim de adequar-se à morbidade e, subseqüentemente, para incluir achados anormais, queixas e circunstâncias sociais que podem ser encontrados no lugar de diagnósticos, em registros relativos à saúde. Pode, portanto, ser usada para classificar registros sob o título de diagnósticos, tanto quanto "razões para admissão" e "razões para consulta" que aparecem em grande variedade de registros dos quais são obtidas as estatísticas de saúde ${ }^{5,8}$.

Cada doença ou condição mórbida tem lugar bem definido na lista de categorias. Consequentemente, ao longo da Classificação há categorias residuais para condições que não podem ser distribuídas em categorias mais específicas. O ideal é que o menor número possível de diagnósticos sejam classificados em categorias residuais ${ }^{8}$.

Alguns estudos realizados no Brasil utilizaram a CID para a classificação das morbidades em Atenção Primária de Saúde - APS ou em inquéritos de saúde. Yaslle Rocha e Nogueira ${ }^{18}$ utilizaram a CID $8^{\text {a }}$ Revisão na descrição de padrões de morbidade em APS através de sistema de "agendamento de consultas”, agrupando-os segundo 17 grupos. A Classificação foi utilizada também em inquérito de saúde realizado por Lebrão e col. ${ }^{19}$, no qual todas as queixas, sintomas, acidentes comuns e diagnósticos referidos, considerados como episódios mórbidos, foram agrupados em capítulos da CID-9. Escuder $^{20}$ usou a Lista de Tabulação para Morbidade da CID-10 para inquérito de morbidade referida e registro de consulta médica de demanda ambulatorial.

Em um inquérito domiciliar realizado na região metropolitana de São Paulo, com a finalidade de descrever o perfil de morbidade e o padrão de uso de serviços de saúde, a CID foi utilizada para codificar as morbidades referidas pelos entrevistados ${ }^{21,22}$. As características do estudo e os resultados obtidos são a seguir descritos.

\section{Metodologia}

Foi selecionada para a pesquisa uma 
amostra populacional probabilística de 10.796 indivíduos, residentes em sete municípios pertencentes à zona oeste da Grande São Paulo*. Houve uma perda de 5,5\% (597) devido à recusa, ausência no domicílio ou outras razões, tendo sido entrevistados 10.199 indivíduos. Os questionários foram aplicados por entrevistadores com segundo grau completo de escolaridade e que receberam treinamento específico para o trabalho de campo. O treinamento enfatizou a importância de registrar as respostas na forma exata como foram expressas pelos entrevistados, evitando qualquer tentativa de "traduzi-las" em linguagem técnica. Os entrevistadores foram acompanhados, avaliados e orientados durante toda a fase do trabalho de campo. Cerca de $10 \%$ das entrevistas realizadas por cada um deles foram avaliadas por meio de uma nova entrevista parcial. As questões foram respondidas diretamente pelos adultos e pelas mães ou pelos responsáveis pelas crianças menores de 14 anos de idade. Em cada domicílio sorteado foram entrevistados grupos específicos definidos a partir do sexo e da idade domínios amostrais, possibilitando, assim, representatividade mesmos dos grupos pouco freqüentes na população como os menores de 1 ano e os idosos. As análises das freqüências e prevalências utilizaram a amostra ponderada pelos inversos das frações amostrais dos domínios.

O perfil de morbidade foi estudado através da referência às doenças ou aos acidentes ocorridos nos quinze dias anteriores à visita domiciliar, e às deficiências físicas e doenças crônicas referidas pelos entrevistados. Destacaram-se, dentre as doenças crônicas, a hipertensão arterial, o diabetes, a convulsão, as doenças cardíacas crônicas, as doenças respiratórias crônicas, "problemas dos nervos”, além de uma questão aberta sobre "outras doenças crônicas". Os acidentes foram especificados de acordo com a natureza do acidente, a natureza do episódio e o local do acidente.
Para a análise, estes dados de morbidade foram inicialmente agrupados pela Classificação Internacional de Doenças, $9^{\mathrm{a}}$ Revisão de $1975^{15}$ (CID-9). Na codificação da morbidade de 15 dias, uma "queixa" ou "sintoma" foi codificado como tal, um diagnóstico referido também foi codificado como tal (exemplos: diarréia, amigdalite, resfriado). Em diversos casos os entrevistados referiram diagnósticos dados por médicos (exemplos: úlcera gástrica, enfisema e outros).

Foi também realizada uma comparação entre a CID-9 e CID-10 reclassificando-se pela CID-10 as morbidades referidas classificadas pela CID-9 como Capítulo XVI (Sintomas, Sinais e Afecções Mal Definidas).

\section{Resultados}

Houve referência a algum problema de saúde, nos quinze dias anteriores à entrevista, para $30,4 \%$ da população; destes, $83,1 \%$ referiram um problema, $13,8 \%$ dois, $2,6 \%$ três e $0,5 \%$ quatro ou mais problemas.

A Tabela 1 mostra a distribuição das morbidades referidas, segundo agrupamentos de causas (capítulos da CID), expressas como prevalência por 1000 pessoas. Considerando o conjunto da população estudada a prevalência maior foi para problemas respiratórios $(99,2 / 1000)$, seguida dos problemas do aparelho digestivo (25,4/1000), de doenças do sistema osteomuscular e tecido conjuntivo (22,9/1000), lesões e envenenamentos (22,6/1000), doenças infecciosas e parasitárias (16,7/1000), doenças do aparelho circulatório (13,6/1000), doenças do sistema nervoso $(12,4 / 1000)$, doenças do aparelho geniturinário $(9,9 / 1000)$ e doenças da pele $(6,5 / 1000)$. Os demais capítulos apresentaram prevalências menores, abaixo de $2 / 1000$. Houve um conjunto de sintomas e sinais referidos, pouco específicos e que foram agrupados no capítulo de sintomas, sinais e afecções mal definidas, correspondendo a uma prevalência de 86,4/1000.

As prevalências de morbidade segundo

* Os sete municípios estudados foram: Taboão da Serra, Embu, Itapecerica da Serra, Juquitiba, Embu-Guaçu, Cotia e Vargem Grande Paulista. 
Tabela 1 - Taxas de prevalência (por 1000) da morbidade referida de 15 dias segundo causas em capítulos da CID-9, faixa etária e sexo.

Table 1 - Prevalence rates (per 1,000) of 15-day referred morbidity according to causes in ICD-9 chapters, age group and gender.

\begin{tabular}{|c|c|c|c|c|c|c|c|c|c|}
\hline \multirow{2}{*}{\multicolumn{2}{|c|}{ Causas }} & \multicolumn{7}{|c|}{ Faixa etária e Sexo } & \multirow{3}{*}{$\begin{array}{r}\text { Todos } \\
16,7\end{array}$} \\
\hline & & \multirow{2}{*}{$\begin{array}{r}<1 \\
30,4\end{array}$} & \multirow{2}{*}{$\begin{array}{r}1 \text { a } 6 \\
47,7\end{array}$} & \multirow{2}{*}{$\begin{array}{r}7 \text { a } 19 \\
13,8\end{array}$} & \multirow{2}{*}{$\begin{array}{r}20-49 \mathrm{M} \\
10,9\end{array}$} & \multirow{2}{*}{$\begin{array}{r}20-49 \mathrm{~F} \\
10,1\end{array}$} & \multirow{2}{*}{$\begin{array}{r}50 \mathrm{e}+\mathrm{M} \\
7,1\end{array}$} & \multirow{2}{*}{$\begin{array}{r}50 \mathrm{e}+\mathrm{F} \\
4,8\end{array}$} & \\
\hline I & - Infecciosas & & & & & & & & \\
\hline II & - Neoplasmas & - & - & - & - & 1,1 & 0,3 & - & 0,3 \\
\hline III & - Endócrino & - & 2,1 & 0,1 & 0,6 & 2,3 & 3,2 & 6,6 & 1,4 \\
\hline IV & - Sangue & - & 3,5 & 3,2 & 0,1 & 1,1 & - & 1,2 & 1,8 \\
\hline V & - Mentais & - & 0,3 & 0,1 & - & 1,1 & 3,1 & 0,2 & 0,5 \\
\hline VI & - Nervoso & 35,4 & 17,1 & 12,6 & 5,6 & 11,6 & 18,7 & 15,5 & 12,4 \\
\hline VII & - Circulatório & - & - & 2,4 & 12,3 & 17,7 & 48,7 & 83,4 & 13,6 \\
\hline VIII & - Respiratório & 275,7 & 166,9 & 86,6 & 68,5 & 93,4 & 67,5 & 95,0 & 99,2 \\
\hline IX & - Digestivo & 8,6 & 7,0 & 18,8 & 30,6 & 37,5 & 35,0 & 38,0 & 25,4 \\
\hline$x$ & - Geniturinário & 2,0 & 1,9 & 5,2 & 3,2 & 28,7 & 5,6 & 12,8 & 9,9 \\
\hline XI & - Gravidez & - & - & 0,8 & - & 0,3 & - & - & 0,4 \\
\hline XII & - Pele & 13,2 & 11,6 & 6,4 & 2,2 & 5,8 & 11,3 & 9,2 & 6,5 \\
\hline XIII & - Osteomuscular & 2,1 & 1,3 & 3,4 & 15,8 & 42,6 & 69,9 & 113,6 & 22,9 \\
\hline XIV & - Congênitas & 1,3 & - & - & - & - & - & - & 0,0 \\
\hline$x V$ & - Perinatal & 4,6 & - & - & - & - & - & - & 0,1 \\
\hline XVI & - Mal definidas & 125,3 & 92,4 & 63,1 & 65,2 & 107,7 & 119,4 & 161,6 & 86,4 \\
\hline XVII & - Lesões & 11,8 & 32,1 & 20,8 & 26,4 & 17,8 & 17,8 & 19,7 & 22,6 \\
\hline
\end{tabular}

os capítulos da CID apresentaram variações quando analisadas por sexo e idade (Tabela 1). Assim, por exemplo, doenças infecciosas e parasitárias, doenças do sistema nervoso e órgãos dos sentidos, doenças do aparelho respiratório e doenças da pele tiveram prevalências mais altas para as idades mais baixas, ocorrendo o inverso com doenças dos aparelhos circulatório, digestivo e geniturinário e do sistema osteomuscular, que apresentaram prevalências mais altas nas idades mais avançadas. Para os demais capítulos as diferenças foram pouco acentuadas.

Com relação à distribuição por sexo as prevalências foram de um modo geral maiores para o sexo feminino comparado com o sexo masculino. Estas diferenças foram mais acentuadas não só para o aparelho geniturinário e o sistema osteomuscular, mas também para as doenças dos aparelhos circulatório e respiratório.

Para o grupo etário "20-49 anos" os valores referidos pelos homens e mulheres foram, respectivamente, para o aparelho geniturinário 3,2/1000 e 28,7/ 1000 e para o sistema osteomuscular 15,8/1000 e 42,6/ 1000.

Com relação ao grupo etário "50 anos e mais" os valores para homens e mulheres foram, para o aparelho geniturinário, 5,6/ 1000 e 12,8/1000 e para o sistema osteomuscular, 69,9/1000 e 113,6/1000.

O número total de "queixas" ou "diagnósticos" foi 4415 , sendo que um mesmo indivíduo pode ter referido mais de uma "queixa" ou "diagnóstico", inclusive classificados em um mesmo capítulo da CID. Estas morbidades referidas distribuíram-se por todos os capítulos da CID-9, conforme pode ser visto na Tabela 2 , que descreve as porcentagens referentes a cada capítulo no total de "queixas" ou "diagnósticos". A maior proporção delas $(30,5 \%)$ foi agrupada no Capítulo XVI - Sintomas, Sinais e Afecções Mal Definidos e as demais 69,5\% distribuíram-se pelos demais capítulos, sendo que 30,1\% foram classificados no Capítulo VIII (Doenças do Aparelho Respiratório), seguido pelo Capítulo IX - Aparelho Digestivo (7,4\%), Capítulo XIII-Osteomuscular (7,1\%), Capítulo VII - Aparelho Circulatório (3,7\%) 
Tabela 2 - Números e percentagens da morbidade referida de 15 dias por causas em capítulos da CID-9 Table 2 - Figures and percentages of 15-day referred morbidity by causes in ICD-9 chapters

\begin{tabular}{|c|c|c|c|}
\hline Capít & tulos & Nos. absolutos & $\%$ na amostra \\
\hline I & - Doenças Infecciosas e Parasitárias & 176 & 4,9 \\
\hline II & - Neoplasmas & 3 & 0,1 \\
\hline III & - Doenças das Glândulas Endócrinas, da Nutrição e & & r \\
\hline IV & - Doenças do Sangue e dos Órgão Hematopoiéticos & 20 & 0,5 \\
\hline V & - Transtornos Mentais & 10 & 0,1 \\
\hline VI & - Doenças do Sistema Nervoso e do Órgãos dos Sentidos & 174 & 3,4 \\
\hline VII & - Doenças do Aparelho Circulatório & 265 & 3,7 \\
\hline VIII & - Doenças do Aparelho Respiratório & 1169 & 30,1 \\
\hline IX & - Doenças do Aparelho Digestivo & 283 & 7,4 \\
\hline$x$ & - Doenças do Aparelho Geniturinário & 130 & 2,9 \\
\hline $\mathrm{XI}$ & - Doenças da Gravidez, do Parto e do Puerpério & 5 & 0,1 \\
\hline XII & - Doenças da Pele e do Tecido Celular Subcutâneo & 92 & 1,9 \\
\hline XIII & - Doenças do Sistema Osteomuscular e do Tecido Conjuntivo & 440 & 7,1 \\
\hline XIV & - Anomalias Congênitas & 1 & 0,0 \\
\hline$x V$ & - Algumas Afecções Originadas no Período Perinatal & 6 & 0,0 \\
\hline $\mathrm{XVI}$ & - Sintomas, Sinais e Afecções Mal definidas & 1403 & 30,5 \\
\hline XVII & - Lesões e Envenenamentos & 211 & 6,6 \\
\hline Total & & 4415 & 100,0 \\
\hline
\end{tabular}

e Capítulo VI - Sistema Nervoso $(3,4)$. Os demais capítulos apresentaram freqüências menores.

As tabelas acima analisadas descrevem, portanto, a primeira delas (Tabela 1) as prevalências das morbidades referidas, agrupadas pelos Capítulos da CID-9, utilizando como denominador o total de entrevistados por sexo e idade; e a segunda (Tabela 2) as porcentagens nos mesmos Capítulos da CID-9, utilizando como denominador o total de morbidades referidas.

Em cada um destes capítulos da CID houve concentração da morbidade referida em algumas patologias específicas, o que pode ser observado no Quadro 1 que descreve os valores referentes aos doze capítulos de maior prevalência (acima de 1 caso por 1000 pessoas), bem como os valores referentes ao capítulo XVI - Sintomas, Sinais e Afecções Mal Definidas.

Em estudos de morbidade referida com períodos recordatórios curtos, até 15 dias, é grande o número de problemas pouco específicos que são referidos, seja porque em alguns casos trata-se de pródromos, seja porque em muitos casos trata-se realmente de sinais ou sintomas que desaparecem sem nenhuma interferência, fazendo parte do processo geral de equilíbrio biológico, sem nenhuma conseqüência aparente. Estes problemas foram classificados no capítulo XVI (Sintomas, Sinais e Afecções Mal Definidas), e os entrevistados que referiram este tipo de queixa o fizeram em alguns poucos sinais $\mathrm{e}$ sintomas, como por exemplo: cefaléia (784.0) com 29,5\%, hipertermia (780.6) com 12,7\%, tosse (786.2) com $11,3 \%$, tontura (780.4) com $6,2 \%$, dor abdominal (789.0) com 5,8\% e náusea e vômito (787.0) com 5,3\%, somando estas queixas $70.8 \%$ deste capítulo.

Com a finalidade de verificar se a CID-10 reduziria a proporção de queixas no capítulo de causas mal definidas, as 1.403 queixas, classificadas pela CID-9 em 51 códigos do Capítulo XVI (Sintomas, Sinais e Afecções Mal Definidas), foram recodificadas de acordo com a CID-10.

Como se pode verificar no Quadro 2, os 51 códigos do Capítulo XVI da CID-9 trans- 
Quadro 1 - Patologias referidas com maior freqüência nos capítulos da Classificação Internacional de Doenças (CID9) que apresentaram taxas de prevalências acima de um caso por mil pessoas.

Chart 1 - Most frequently referred conditions of chapters of the International Classification of Diseases (ICD-9) with prevalence rates above one case per thousand.

\begin{tabular}{lrr}
\hline & $N^{\circ 1}$ & $\%^{2}$ \\
\hline Capítulo I - Doenças infecciosas e parasitárias & & \\
Infecções intestinais (001-009) & $(94)$ & 49,7 \\
Helmintíases (120-129) & $(32)$ & 17,4 \\
Varicela (052) & $(15)$ & 10,4 \\
Parotidite epidêmica (072) & $(10)$ & 6,0 \\
Rubéola (056) & $(5)$ & 4,1 \\
Escabiose (133.0) & $(5)$ & 2,0 \\
Subtotal & $(161)$ & 89,6 \\
Todas as demais & $(15)$ & 10,4 \\
Total & $(176)$ & 100,0 \\
\hline
\end{tabular}

Capítulo III - Doenças das glândulas endócrinas, da nutrição e do metabolismo e transtornos imunitários

Diabetes mellitus (250)

Desidratação (276.5)

Subtotal

Todas as demais

(15) 68,3

(6) 24,0

(21) 92,3

(6) 7,7

Total

Capítulo IV - Doenças do sangue e dos órgãos

hematopoiéticos

Anemias (285)

Todas as demais

Total

(27) 100,0

Capitulo VI - Doenças do sistema nervoso e dos órgãos dos sentidos

Otalgia (388.7)

Transtornos da conjuntiva (372)

Labirintite (386.3)

Outros transtornos do olho (379)

Otite média (382.9)

Enxaqueca (346)

Subtotal

Todas as demais

Total

(18) 74,2

(2) 25,8

(20) 100,0

Capítulo VII - Doenças do aparelho circulatório

Hipertensão essencial (401)

Hipotensão (458)

Varizes das extremidades inferiores (454)

Doença do coração não especificada (429.9)

Hemorróidas (455)

Doenças cerebrovasculares (430-438)

Subtotal

(45) 34,8

(19) 12,5

(21) 12,0

(19) 9,8

(15) 7,6

(15) 5,3

(134) 82,0

(40) 18,0

(174) 100,0

Todas as demais

Total

Capítulo VIII - Doenças do aparelho respiratório

Gripe (487)

Resfriado comum (460)

Faringite aguda (462)

Bronquite (490)

Pneumonia (486)

Sinusite crônica (473)

Outra doenças da faringe (478)

Rinite alérgica (477)

Subtotal

Todas as demais

(167) 63,6

(15) 9,0

(33) 8,5

(10) 3,1

(7) 2,1

(9) 1,9

(241) 88,2

(24) 11,8

(265) 100,0

(632) 47,2

(242) 23,1

(109) 13,2

(92) $\quad 7,4$

(26) 2,0

(19) 1,3

(13) 1,3

(7) 1,2

(1140) 96,7

(29) 3,3

Total

Capítulo IX - Doenças do aparelho digestivo

Transtornos da função gástrica (536)

Afecções dos dentes e de suas estruturas de

sustentação (520-525)

(1169) 100,0

Úlcera gástrica (531)

Constipação (564.0)

Transtornos do fígado não especificados (573)

Hérnia da cavidade abdominal (550-553)

Subtotal

Todas as demais

Total

(109) 42,4

(36) 14,5

(14) 7,1

(23) 5,4

(16) 5,1

(17) 4,7

(20) 3,9

(235) 83,1

(48) 16,9

(283) 100,0

\begin{tabular}{lrr}
\hline & $N^{\circ 1}$ & $\%^{2}$ \\
\hline $\begin{array}{lr}\text { Capítulo X - Doenças do aparelho geniturinário } \\
\text { Outros transtornos do rim e do ureter(593) }\end{array}$ & $(17)$ & 14,4 \\
$\begin{array}{l}\text { Síndrome de tensão pré-menstrual (625.4) } \\
\text { Sinais e sintomas da mama (611.7) }\end{array}$ & $(21)$ & 12,4 \\
$\begin{array}{l}\text { Transtornos da menstruação e outras hemorragias } \\
\text { anormais do trato genital feminino (626) }\end{array}$ & $(7)$ & 11,6 \\
$\begin{array}{l}\text { Infecção do trato urinário de localização } \\
\text { não especificada (599.0) }\end{array}$ & (13) & 9,3 \\
$\begin{array}{l}\text { Doenças inflamatórias dos órgãos pélvicos } \\
\text { femininos (614-616) }\end{array}$ & $(16)$ & 8,4 \\
$\begin{array}{l}\text { Dor e outros sintomas associados com os órgãos } \\
\text { genitais não especificados (625.9) }\end{array}$ & $(7)$ & 4,7 \\
$\begin{array}{l}\text { Cistite (545.9) } \\
\text { Subtotal }\end{array}$ & $(10)$ & 1,7 \\
Todas as demais & $(86)$ & 72,4 \\
Total & $(32)$ & 27,6 \\
\hline Capitulo XII - Don & $(130)$ & 100,0 \\
\hline
\end{tabular}

Capítulo XII - Doenças da pele e do tecido celular subcutâneo

Outros transtornos da pele e do tecido

celular subcutâneo (709) (25) 41,7

$\begin{array}{ll}\text { Prurido e afecções afins (698) } & \text { (28) } 23,7\end{array}$

Infecções da pele e do tecido celular

subcutâneo (680-686)

Úlcera crônica da pele (707)

Dermatite de contato e outros eczemas (692)

(11) 15,7

Subtotal

Todas as demais

(15) 7,1

(86) 91,2

Total

(6) $\quad 8,8$

Capítulo XIII - Doenças do sistema osteomuscular e do

tecido conjuntivo

Dor nas costas não especificada (724.5) (135) 34,8

$\begin{array}{lll}\text { Dor nos membros (729.5) } & \text { (132) } 26,7\end{array}$

Transtornos das costas não especificados (724.9) (31) $\quad 10,4$

Reumatismo não especificado e fibrosite (729.0) (61) $\quad 7,5$

Artropatias e transtornos afins (710-719)

Cervicalgia e torcicolo (723.1 e 723.5) (12) 4,6

Outros sintomas relativos aos membros (729.8) (10) 1,8

Dor na coluna vertebral torácica (724.1) (8) 1,2

Lumbago (724.2) (12) 1,1

Subtotal (418) 93,0

Todas as demais $\quad$ (22) 7,0

Total (440) 100,0

Capítulo XVI Sintomas, sinais e afecções mal definidas

Cefaléia (784.0) (367) 29,5

$\begin{array}{ll}\text { Hipertermia (780.6) } & \text { (167) } 12,7\end{array}$

Tosse (786.2) (161) 11,3

Tontura (780.4) (98) 6,2

Dor abdominal (789.0) (90) 5,8

Náusea e vômito (787.0) (57) 5,3

Dispnéia (786.0) (64) 4,5

Dor de garganta (784.1) (42) 3,5

Dor torácica (786.5) (46) 2,4

Mal estar e fadiga (780.7)

Subtotal (1123) 83,0

Todas as demais (280) 17,0

Total (1403) 100,0

Capítulo XVII Lesões e envenenamentos

Alergia não especificada (995.3) (66) 28,5

Ferimentos (870-897)

Algumas complicações traumáticas e

traumatismos não especificados (958-959) (28) 13,3

Fraturas (800-829) (24) 11,1

Subtotal (168) 77,7

Todas as demais (43) 22,3

Total

(211) 100,0

Números absolutos na amostra

${ }^{2}$ Porcentagens na amostra ponderada. 
Quadro 2 - Sintomas, sinais e afecções mal definidas na morbidade referida de 15 dias, codificados pela CID-9 e pela CID-10.

Chart 2 - Symptoms, signs and ill-defined conditions in 15-day referred morbidity, coded by ICD-9 and ICD-10.

\begin{tabular}{|c|c|c|c|}
\hline CID 9 & $\mathrm{~N}^{\circ}$ & CID 10 & $\mathrm{~N}^{\circ}$ \\
\hline \multirow[t]{2}{*}{$780.0^{*}$} & 2 & R40.0 & 1 \\
\hline & & R40.2 & 1 \\
\hline 780.2 & 5 & R55 & 5 \\
\hline $780.3^{*}$ & 1 & R56.8 & 1 \\
\hline 780.4 & 97 & $\mathrm{R} 42$ & 97 \\
\hline \multirow[t]{2}{*}{780.5} & 11 & G47.0 & 9 \\
\hline & & $\mathrm{R} 40.0$ & 2 \\
\hline $780.6^{*}$ & 172 & R50.9 & 172 \\
\hline 780.7 & 31 & R53 & 31 \\
\hline \multirow[t]{3}{*}{780.9} & 45 & R41.3 & 3 \\
\hline & & R52.9 & 41 \\
\hline & & R68.0 & 1 \\
\hline $781.0^{*}$ & 2 & $\mathrm{R} 25.1$ & 2 \\
\hline 781.9 & 1 & M62.9 & 1 \\
\hline $782.0^{*}$ & 26 & $\mathrm{R} 20.2$ & 26 \\
\hline 782.1 & 1 & L53.9 & 1 \\
\hline \multirow[t]{4}{*}{$782.2^{*}$} & 7 & L29.9 & 1 \\
\hline & & $\mathrm{R} 22.0$ & 1 \\
\hline & & $\mathrm{R} 22.7$ & 1 \\
\hline & & R22.9 & 4 \\
\hline \multirow[t]{2}{*}{$782.3^{*}$} & 17 & R60.0 & 15 \\
\hline & & R60.9 & 2 \\
\hline 782.6 & 1 & R23.1 & 1 \\
\hline 782.7 & 1 & R23.3 & 1 \\
\hline \multirow[t]{2}{*}{782.9} & 11 & $\mathrm{R} 21$ & 5 \\
\hline & & R23.8 & 6 \\
\hline 783.0 & 5 & R63.0 & 5 \\
\hline 784.0 & 361 & R51 & 361 \\
\hline 784.1 & 43 & R07.0 & 43 \\
\hline \multirow[t]{2}{*}{$784.4^{*}$} & 6 & R49.0 & 5 \\
\hline & & R49.1 & 1 \\
\hline $784.5^{*}$ & 1 & R47.8 & 1 \\
\hline 784.9 & 6 & R68.8 & 6 \\
\hline 785.0 & 8 & R00.0 & 8 \\
\hline 785.1 & 1 & $\mathrm{R} 00.2$ & 1 \\
\hline
\end{tabular}

\begin{tabular}{|c|c|c|c|}
\hline CID 9 & $\mathrm{~N}^{\circ}$ & CID 10 & $\mathrm{~N}^{\circ}$ \\
\hline 785.2 & 3 & R01.1 & 3 \\
\hline 785.9 & 2 & R09.8 & 2 \\
\hline \multirow[t]{2}{*}{$786.0^{*}$} & 64 & R06.0 & 4 \\
\hline & & R06.8 & 60 \\
\hline 786.2 & 161 & R05 & 161 \\
\hline 786.3 & 4 & R04.0 & 4 \\
\hline 786.4 & 4 & J00 & 4 \\
\hline \multirow[t]{3}{*}{$786.5^{*}$} & 46 & R07.2 & 6 \\
\hline & & R07.3 & 36 \\
\hline & & R07.4 & 4 \\
\hline 786.7 & 6 & R09.8 & 6 \\
\hline \multirow[t]{3}{*}{786.9} & 3 & R06.0 & 1 \\
\hline & & R09.8 & 1 \\
\hline & & R68.8 & 1 \\
\hline 787.0 & 58 & $\mathrm{R} 11$ & 58 \\
\hline 787.1 & 7 & $\mathrm{R} 12$ & 7 \\
\hline 787.3 & 4 & $\mathrm{R} 14$ & 4 \\
\hline 787.7 & 1 & R19.5 & 1 \\
\hline 787.9 & 3 & R19.8 & 2 \\
\hline R68.2 & 1 & & \\
\hline 788.0 & 26 & N23 & 26 \\
\hline 788.3 & 5 & R32 & 5 \\
\hline 788.4 & 1 & R35 & 1 \\
\hline $788.6^{*}$ & 1 & R39.1 & 1 \\
\hline \multirow[t]{3}{*}{$788.9^{*}$} & 18 & N39.9 & 3 \\
\hline & & R30.9 & 4 \\
\hline & & R39.8 & 11 \\
\hline $789.0^{*}$ & 90 & R10.4 & 90 \\
\hline 789.2 & 1 & R16.1 & 1 \\
\hline \multirow[t]{2}{*}{$789.9^{*}$} & 5 & M54.5 & 4 \\
\hline & & R52.9 & 1 \\
\hline 796.4 & 2 & R09.8 & 1 \\
\hline 799.2 & 7 & R45.0 & 7 \\
\hline 799.3 & 17 & R53 & 17 \\
\hline 799.8 & 1 & R68.8 & 01 \\
\hline Total & 1403 & & $1402^{(1)}$ \\
\hline
\end{tabular}

Notas: (1) Excluída 1 queixa de classificação ignorada.

Em negrito, as queixas excluídas do capítulo sintomas, sinais e afecções mal definidas, após classificação pela CID-10.

(*) Receberam categorias próprias na CID-10

Notes: ${ }^{(1)}$ Excluded 1 complaint of unspecified classification.

In bold, are the complaints excluded from the symptoms, signs and ill-defined conditions chapter, after classification by ICD-10. (*) Received own categories in ICD-10

formaram-se em 70 códigos na CID-10, sendo que 8 passaram a pertencer a outro capítulo e, dentre os que permaneceram no capítulo, 19 encontram-se como condição re- sidual (códigos .8 ou .9). Portanto, cerca de $70 \%$ (43) das queixas pertencentes ao Capítulo XVIII da CID-10 (Sintomas, Sinais e Achados Anormais de Exames Clínicos e de La- 
boratório não Classificados em Outra Parte) caracterizaram-se como sintomas gerais para os quais foi possível maior detalhamento dentro do próprio capítulo.

É importante considerar que algumas destas condições residuais representam ganho de especificidade se comparadas à CID9, pois pertencem a novas categorias instituídas na última classificação, aumentando a possibilidade de detalhamento das queixas. Foi o que ocorreu com o código R50.9 (febre não especificada), pois a febre de origem desconhecida (subcategoria 780.6) passou a ter uma categoria própria (R50).

Observando cada caso após a reclassificação, verificou-se que ocorreram situações de aumento e de manutenção da especificidade. Algumas delas merecem destaque:

- mudança de 8 queixas (em negrito no Quadro 2) do capítulo Sintomas, Sinais e Afecções Mal Definidas para outro capítulo, o que caracteriza ganho de especificidade;

- queixas que pertenciam a categorias gerais na CID-9, receberam categorias próprias na CID-10 (marcadas por* no Quadro 2) e apresentam maior detalhamento em suas subcategorias. Podem ser citadas como exemplo, as queixas classificadas em 780.0 (subcategoria coma e estupor) que com a criação de uma categoria própria para sonolência, estupor e coma (R40), foram codificadas em duas subcategorias: R40.0 (sonolência) e R40.2 (coma não especificado);

- queixas classificadas em 2 subcategorias na CID-9: 780.7 (mal estar e fadiga) e 799.3 (debilidade não especificada), com a CID10 passaram a pertencer a uma única categoria R53 (mal estar e fadiga) que incluiu a debilidade; o que poderia ser perda de especificidade, deixa de sê-lo ao se considerar a semelhança das queixas.

Diante de pelo menos uma das seguintes alterações, admitiu-se que houve ganho de especificidade:

- de uma subcategoria na CID-9 para uma categoria na CID-10, com possibilidade de subcategorias;
- de condição residual (códigos .8 ou .9) para outra subcategoria diferente de residual (códigos de .0 a .7);

- de uma única subcategoria para várias subcategorias que permitiam maior detalhamento;

- do capítulo Sintomas, Sinais e Afecções Mal Definidas para outro capítulo.

Houve manutenção da condição quando o código apresentou a mesma especificidade em ambas as Classificações.

Dos 51 códigos do capítulo Sintomas, Sinais e Afecções Mal Definidas da CID-9, 24 ganharam especificidade e 27 permaneceram na mesma condição após a classificação pela CID-10. Os resultados encontrados com a comparação demonstram que, para a maioria dos problemas referidos neste inquérito, a CID-10 foi melhor para codificar condições mal definidas, pois possibilitou aumento na especificidade, seja por mudanças que ocorreram para códigos mais detalhados internamente ao próprio capítulo, seja por transferência para outros capítulos e conseqüente redução do número de queixas no capítulo de causas mal definidas.

\section{Considerações Finais}

Os inquéritos de saúde de base populacional apresentam algumas peculiaridades. A primeira delas é em relação aos problemas de saúde referidos nos quinze dias anteriores à entrevista. Deve ser ressaltado que a grande vantagem deste tipo de estudo é conhecer todo o espectro de afecções ocorridas, desde aquelas muito bem definidas, eventualmente com diagnóstico clínico e laboratorial, até os sintomas ou sinais pouco característicos, que eventualmente desapareceram com automedicação ou mesmo sem nenhuma interferência. Isto leva a uma freqüência de "sintomas, sinais e afecções mal definidas" maior do que a encontrada em estudos de morbidade da população atendida pelos serviços de saúde, a qual representa uma seleção do total de problemas ocorridos na população. A segunda peculiaridade é que a referência aos problemas de saúde pode ser feita com terminologia não pertencente à 
nosologia médica, levando à necessidade de adequação dos termos à nosografia médica.

Estes fatos ocorrem em proporção muito menor para as doenças crônicas, pois muito do que é referido baseia-se em diagnóstico médico e, praticamente inexistem para as deficiências físicas.

Apesar das características próprias dos inquéritos de base populacional é bastante satisfatória a classificação das morbidades referidas pelos entrevistados por meio da

CID. Além disto, a CID-10 apresenta vantagens em relação a CID-9, no caso dos inquéritos: possibilidade de classificar subcategorias residuais ao longo dos diferentes capítulos e maior especificidade do Capítulo XVIII (Sintomas, Sinais e Achados Anormais de Exames Clínicos e de Laboratórios não Classificados em Outra Parte) com definições mais precisas das categorias e subcategorias.

\section{Referências}

1. WONCA. An international classification of health problems of primary care:. working party report. London: The Devonshire Press; 1976. (Occasional Paper, 1).

2. White KL. Health surveys: who, why and what? World Health Stat Q 1985; 38:2-14.

3. Laurenti R. Análise da informação em saúde: 18931993, cem anos de classificação internacional de doenças. Rev Saúde Pública 1991; 25:407-17.

4. Lebrão ML. Estudos de morbidade. São Paulo: EDUSP;1997.

5. Organização Mundial da Saúde. Classificação estatística internacional de doenças e problemas relacionados à saúde; $10^{a}$ revisão. São Paulo: EDUSP; 1994. v.2.

6. Wells R. Experiences with the international classification of diseases and emerging problems. In: International Conference on Health Statistics for the Year 2000; 1982 Sept 27; Bellagio, Italy. Budapest: Statistical Publishing House/WHO; 1984. p.18-33.

7. Last JM. Nosography: conceptual, epidemiological and statistical implications. In: International Conference on Health Statistics for the Year 2000; 1982 Sept 27; Bellagio, Italy. Budapest: Statistical Publishing House/ WHO; 1984. p.34-48.

8. Gersenovic M. The ICD family of classifications. Methods Inf Med 1995; 34:172-5.

9. Bensten BG. Classifying of health problems in primary medical care. In: WONCA. An international classification of health problems of primary care. London: The Devonshire Press; 1976. p.3-9. (Occasional Paper, 1).
10. Meads S. La classificación de la OMS por "razones de la consulta”. Cronica OMS 1983; 37: 193-6.

11. Laurenti R. Lay reporting of health and morbidity problems. In: International Conference on Health Statistics for the Year 2000; 1982 Sept 27; Bellagio, Italy. Budapest: Statistical Publishing House/WHO; 1984. p. 99-120.

12. White KL. Introduction to health statistics for the year 2000. In: International Conference on Health Statistics for the Year 2000; 1982 Sept 27; Bellagio, Italy. Budapest: Statistical Publishing House/WHO; 1984. p.12-7.

13. Wood M. Evaluation of primary care classifications and the potential impact of the reason for encounter classification. In: International Conference on Health Statistics for the Year 2000; 1982 Sept 27; Bellagio, Italy. Budapest: Statistical Publishing House/WHO; 1984. p.49-89.

14. Lebrão ML. Classificação internacional de motivos de consulta para assistência primária: teste em algumas áreas brasileiras. Rev Saúde Pública 1985; 19:69-78.

15. Organização Mundial da Saúde. Manual de classificação internacional de doenças, lesões e causas de óbitos; $9^{a}$ revisão 1975. São Paulo: Centro da OMS para Classificação de Doenças em Português; 1978.

16. Organização Mundial da Saúde. Classificação estatística internacional de doenças e problemas relacionados à saúde; $10^{a}$ revisão. $2^{\text {a }}$ ed. São Paulo: EDUSP; 1995. v.1.

17. Avilan Rovira JM. La classificación internacional de enfermidades en la formación de analistas de información. Cuad Esc Salud Publica 1989; 53: 3-14. 
18. Yaslle Rocha JS, Nogueira JL. Padrões de morbidade em assistência primária na região de Ribeirão Preto, SP (Brasil). Rev Saúde Pública 1985; 19: 215-24.

19. Lebrão ML, Carandina L, Magaldi C. Análise das condições de saúde e de vida da população urbana de Botucatu, São Paulo (Brasil). IV - Morbidade referida em entrevistas domiciliárias, 1983-1984. Rev Saúde Pública 1991; 25: 452-60.

20. Escuder MML. Morbidade ambulatorial como expressão das necessidades de saúde da população [Dissertação de Mestrado]. São Paulo: Faculdade de Saúde Pública da USP; 1998.
21. Cesar CLG, Figueiredo GM, Westphal MF, Cardoso MRA, Costa MZA, Gattás VL. Morbidade referida e utilização de serviços de saúde em localidades urbanas brasileiras: metodologia. Rev Saúde Pública 1996; 30:153-60.

22. Cesar CLG. Morbidade referida e utilização de serviços de saúde na Grande São Paulo. O perfil da desigualdade em saúde [Tese de Livre Docência]. São Paulo: Faculdade de Saúde Pública da USP;1997. 